\title{
Establishing a clinical database for hospital attendances because of self-harm
}

Self-harm is a major risk factor for suicide (Gunnell \& Frankel, 1994) with around a quarter of suicides preceded by non-fatal self-harm in the previous year (Owens \& House, 1994).
Strategies for suicide prevention should include accurate monitoring of health service contacts due to selfharm. Unfortunately, the published literature points to few practical steps for ensuring this accuracy. We offer an account of running a database, to assist others who might be setting out on this monitoring process.

\section{Planning the data collection}

Leeds is an industrial and commercial city of about 750000 people in the north of England, served by two large teaching hospitals. In recent studies of the epidemiology of self-harm (Horrocks et al, 2002, 2003), we aimed to record details of all those who attended hospital because of non-fatal self-harm over an 18-month period, regardless of the stage at which they left the hospital.

\section{Deciding on the point of case ascertainment}

In the published epidemiology of hospital contact after self-harm, cases have been ascertained in a variety of settings: specialist mental health services; general hospital wards; accident and emergency attendances; or combinations of these (Evans et al, 1996). To determine the number of people attending hospital because of selfharm in Leeds, we chose accident and emergency as our point of ascertainment for two reasons. First, it has been established that most people who present to health services after self-harm either attend accident and emergency as their first point of contact or they are referred there (Crawford \& Wessely, 1998). Second, the evidence is clear that a high proportion of those attending accident and emergency are discharged home directly, often without any specialist mental health service contact (Owens, 1990; Kapur et al 1998).

We defined attendance at accident and emergency as an occasion when the person had stayed long enough for their details to be recorded by a clerk and for a record card to be produced.

\section{Defining self-harm}

An attendance because of self-harm was one in which the patient reported harm of any sort as being selfinflicted, or in which a clinician deemed the harm to be self-inflicted. The definition applied easily to many cases of self-poisoning by ingestion and by inhalation of carbon monoxide, and to self-injuries such as lacerations, multiple injuries from jumping, strangulation, asphyxiation and gunshots. More difficult were 'rescues' from an attempt - people about to jump off a bridge or retrieved from the middle of a busy road; we decided to classify these few attendances as self-harm even though no physical harm had occurred. We excluded people who attended accident and emergency simply because they reported feeling suicidal. We included cases where people had punched walls or deliberately put their hand through glass; these cases were recorded as 'probable' self-harm so that we could include or exclude them in analyses. We included harm arising from recreational use of drugs only where it was clear that the person had intentionally taken an excess to cause harm.

\section{Identifying episodes of self-harm}

Clerical staff in the accident and emergency reception area record patients' reasons for attending and basic personal information - obtained from each patient, or from accompanying friends or relatives or ambulance crew. We received a printed list of relevant attendances regularly from each accident and emergency department. The two local accident and emergency departments used different computer systems and have different methods of coding attending at, and discharge from, accident and emergency. The codes that comprised our regular reports therefore differed at each site and were approximately as follows: Hospital 1 - all reasons for attending coded as 'deliberate self-harm' or 'psychiatric' or 'did not wait for examinations'; or diagnosis coded as 'poisoning/overdose'; or method of departure coded as 'left before treatment'. Hospital 2 - all reasons for attending coded as 'deliberate self-harm' or 'psychiatric'; or booking-in code of 'deliberate self-harm' or 'overdose/poisoning' or 'mental illness' or 'behaving strangely' or 'appears drunk'.

Therefore at Hospital 1, we only used codes applied at entry to the accident and emergency department, whereas at Hospital 2 we used codes at entry and exit from the department. We collected these reports twice weekly at each hospital for all people aged 12 years or over, and we used them to locate each clinical case record in the accident and emergency department in order to ascertain whether the attendance was for self-harm.

\section{Identifying individuals who attend more than once}

To make the distinction between episodes and separate people, we completed two research data sheets for every 
(8)

special articles attendance. On the first sheet we recorded patient identity information and two study numbers; one for the episode of self-harm and the other to identify the individual person. As we added new episodes to the database, each was checked against existing names. Using this method we were able, from the point of first entry of data, to keep patients' names away from all demographic and clinical data about episodes - stored separately in a password-protected document. We routinely used a laptop computer for collection of data so that we could allocate identity numbers, check accurately for previous attendances of self-harm and anonymise data at the site and point of collection. At the end of the 18-month study period, we again checked all names and dates of birth to ensure that each person had only one unique identity number and that all episode identity numbers were different so that no episode was entered twice.

\section{Additional methods for identifying episodes of self-harm}

The above method does not identify all self-harm attendances because of inconsistent coding of episodes or diagnoses and delay in entering information on the accident and emergency computer systems. We therefore used a number of additional methods to identify retrospectively these 'missed' attendances.

First, we printed a monthly report and thereby identified attendances that did not appear on the twiceweekly reports. Second, we examined at each hospital the mental health service's records of referral from accident and emergency or in-patient wards. Telephoned referrals were recorded in a single ledger, which the researcher cross-checked once a month, returning to accident and emergency to locate the clinical record when an additional case was found. This ascertainment of otherwise missing cases was impeded in two ways: inconsistent recording of out-of-hours referrals by the mental health services; and our access only to referrals to the service for working-age adults but not those for older adults or children.

Clinical records were often missing from accident and emergency: we created a separate database for them and the researcher re-checked for missing records once a week. At the end of the study period, we carried out a laborious final check of subsequent attendances, in the hope of finding the record in question, stored alongside the person's later records.

\section{What happened in practice}

\section{Definition of self-harm}

The researcher could not always easily determine whether an attendance was due to self-harm. There was particular difficulty when notes were incomplete and when recreational drugs had been taken in large amounts. On these occasions, the researcher had to use judgement based on the amount of drugs taken, the context of the overdose and any existing study data about that person's history of self-harm.

\section{Identifying episodes of self-harm}

We identified 5066 episodes of self-harm at the two accident and emergency departments in 18 months, undertaken by 3239 people (Horrocks et al, 2002). With an episode-to-people ratio of 1.6, identification of reattendance is a major task, impeded by factors such as: errors in the recording of names, addresses and dates of birth; people sharing the same name; names changed after marriage; and the occasional use of pseudonyms The researchers had to develop methodical procedures for decision making. Where there was persisting doubt about attributing new episodes to an existing person, two of the research team made a joint decision.

Many episodes in the final list were derived not from the accident and emergency printed lists but from the additional steps outlined above. For example, at one of our hospitals 120 out of 2644 (4.5\%) attendances were identified using the post-dated monthly reports, and 532 out of $2644(20 \%)$ attendances were identified from the ledger that recorded psychiatric referrals. For a further 71 episodes (spread across the two hospitals) identified

Box 1. A checklist for establishing a clinical database of self-harm attendances at accident and emergency

- Decide a clear working definition of self-harm with inclusion and exclusion criteria

- Provide researchers with a written protocol of which data are to be collected, and a data extraction sheet

- Ensure that all researchers not employed by the health service have contracts (such as honorary hospital contracts) that allow them access to patient records

- Use wide search criteria at accident and emergency to identify episodes of self-harm

- Revisit accident and emergency records weekly and every 6 months to check for previously missing records

- Use the accident and emergency computer system to check for re-attendances and then search to see if missing records have been collated with subsequent attendances

- Establish project rules for dealing with inconsistencies in the recording of names or dates of birth

- Cross-check episodes identified through accident and emergency with other sources such as referrals to hospital-based mental health services

- Carry out regular checks of names, dates of birth and dates of attendance to ensure that people are correctly identified by their identity number and that pseudonyms are recorded

- Ensure that there is a system in place for identifying people who re-attend accident and emergency after self-harm

- Document the number of missing records and unobtainable information

- Clean data at regular intervals, and at the end of the project, to ensure that no two episodes are the same and that people are accurately distinguishable from episodes

- Calculate inter- and intra-rater reliability of identification of self-harm episodes and coding of data

- Take adequate steps to comply with local legislation on the keeping of confidential information and ensure that data on individuals are kept separately from records of their names and identifying codes 
through the psychiatry ledger, we could find no corresponding record on the accident and emergency computer system for that person and date, even after trying alternative spellings and wider searches of names and dates. Some might have arisen from direct transfer of psychiatric in-patients to medical wards, but most were probably a consequence of inaccuracy in the giving or recording of biographical information.

In the end, we could not locate 440 accident and emergency case records. One hundred and twenty-eight of these were likely to have been episodes of self-harm because they were recorded as such in the psychiatric ledger, but they could not be included because we could not find demographic and clinical information. An unknown proportion of the other missing records were also likely to have represented episodes of self-harm.

\section{Resources}

We had one full-time research assistant and one parttime clerical assistant; this was barely adequate for the task. We believe that two full-time data collectors would be preferable for a database of this size - to allow for analysis of data. The provision of a comprehensive protocol, together with training at the start of the study and good supervision, should be adequate preparation for research staff not experienced in this area of work.

\section{Concluding remarks}

Most of the difficulties we encountered were not related directly to self-harm but were consequences of the administrative systems of large hospitals, where the volume and speed of information collection leads to inaccuracy. Our experience points to the need to incorporate cross-checking procedures. Finally, we have set out a checklist of points for consideration when establishing a clinical database of self-harm attendances to hospital (Box 1)

\section{Declaration of interest}

None.

\section{References}

CRAWFORD, M. \& WESSELY, S. (1998)

The changing epidemiology of deliberate self harm - implications for accident and emergency department: a service provision. Health Trends, $\mathbf{3 0}$ 66-68.

clinical database study. British Journal of Psychiatry, 183, 34-39.

EVANS, J., JOHNSON, C., STANTON, R. et al (1996) How to establish case registers: 2. Non-fatal deliberate self-harm. Psychiatric Bulletin, 20, 403-405.

GUNNELL, D. \& FRANKEL, S. (1994)

Prevention of suicide: aspirations and evidence. BMJ, 308, 1227-1233.

HORROCKS, J., HOUSE, A. \& OWENS, D. (2002) Attendances in the Accident and Emergency Department Following SelfHarm: a Descriptive Study. Leeds: University of Leeds.

KAPUR, N., HOUSE, A., CREED, F., et al (1998) Management of deliberate self poisoning in adults in four teaching hospitals. BMJ, 316, 831-832.

OWENS, D. (1990) Self-harm patients not admitted to hospital. (1990) Journal of the Royal College of Physicians of London, 24, 281-283.

OWENS, D., HOUSE, A. (1994) General hospital services for deliberate self-

harm: haphazard clinical provision, little research, no central strategy. Journal of the Royal College of Physicians of London, 28, 370-371.

Judith Horrocks Research Psychologist, Allan House Professor of Liaison Psychiatry, *David Owens Senior Lecturer in Psychiatry, Academic Unit of Psychiatry and Behavioural Sciences, 15 HydeTerrace, Leeds LS2 9LT 